\title{
Respostas emocionais da variação linguística: Análise exploratória de rastreio ocular
}

\author{
Raquel Meister Ko. Freitag ${ }^{1}$, Julian Tejada ${ }^{2}$, René Alain Santana de Almeida ${ }^{1}$, \\ Paloma Batista Cardoso ${ }^{3}$, Victor Renê Andrade Souza ${ }^{3}$, Vanesca Carvalho Leal ${ }^{3}$ \\ ${ }^{1}$ Departamento de Letras Vernáculas - Universidade Federal de Sergipe (UFS) \\ Cidade Universitária - 49.100-000 - São Cristóvão - SE - Brazil \\ ${ }^{2}$ Departamento de Psicologia - Universidade Federal de Sergipe \\ ${ }^{3}$ Programa de Pós-Graduação em Letras - Universidade Federal de Sergipe \\ \{rkofreitag, jtejada, rene, palomabatista, victor.rene, vanescaleal\}@ academico.ufs.br
}

\begin{abstract}
An exploratory study of exposure of participants to variants of a socially salient linguistic variable, progressive palatalization, using eye tracking is presented to examine the processing of linguistic variation. The results show that exposure to the stigmatized variant captured participants' attention and increased participants' pupil dilation, which can be interpreted as evidence of an emotional response.
\end{abstract}

Resumo. Um estudo exploratório de exposição de participantes às variantes de uma variável linguística saliente do ponto de vista social, a palatalização progressiva, foi realizado com o uso de rastreamento ocular, para examinar o processamento da variação linguística. Os resultados mostram que exposição à variante estigmatizada captou a atenção e aumentou a dilatação da pupila dos participantes, o que pode ser interpretado como evidência de uma resposta emocional.

\section{Introdução}

Na observação de processos variáveis nas línguas, o papel do processamento tem ganhado relevo nos últimos anos, especialmente por conta da difusão de recursos tecnológicos não invasivos que permitem observar os efeitos de uma certa realização linguística não esperada para o contexto, em termos de respostas emocionais e demanda de atenção.

Uma das maneiras de observar se uma estrutura é mais saliente do que outra é quanto ao dispêndio de esforço de processamento. Na abordagem sociolinguística, no entanto, nem sempre formas salientes do ponto de vista cognitivo e de frequência são necessariamente salientes do ponto de vista social (e vice-versa), o que torna a mensuração do efeito de saliência muito difícil [Kerswill e Williams 2011, Kecskes 2011, Boswijk et al. 2020].

Diferentes abordagens têm sido utilizadas para observar efeitos de saliência no processo de variação linguística: estrutural, distribucional e sociocognitiva [Freitag 2018]. Os efeitos da estrutura linguística, como a saliência fônica e sua relação com a concordância, por exemplo, já são amplamente descritos no português. A saliência distribucional, implementada pelo monitor sociolinguístico [Labov et al. 2006, 
Labov et al. 2011, Levon e Fox 2014, Freitag 2020], constructo para aferir aspectos perceptuais da variação linguística quantitativa em abordagens experimentais, como os estudos de percepção sociolinguística, tem revelado o quão sensíveis os falantes são às diferenças de frequência de uso de uma mesma variante.

O nível de consciência social desempenha um importante papel ao determinar quais variantes são sujeitas à correção [Labov 1972], e envolve o conhecimento implícito e explícito sobre a língua: perceber, reconhecer e processar a variação [Squires 2016]. Processos cognitivos ativados por um gatilho, como um traço sociolinguístico, ainda que controlados conscientemente, deixam pistas: a dilatação da pupila ou as expressões faciais [Freitag et al. 2020] também podem ser consideradas como evidências do julgamento social subjacente, que é a matriz do preconceito linguístico. Para a percepção sociolinguística, os efeitos da saliência sociocognitiva podem ser medidos na relação entre a saliência estrutural e a diferença na distribuição de variáveis que carregam indexação social e as que não carregam indexação social. Sendo um universal, os efeitos de superfície da saliência cognitiva apresentam um correlato fisiológico, um deles as respostas emocionais.

Emoções são respostas fisiológicas automáticas (como a dilatação da pupila [Partala e Surakka 2003]) ante determinados tipos de estímulos, e sensações são as experiências conscientes que acompanham essas mudanças fisiológicas. Estudos experimentais têm mostrado que as mudanças de excitação fisiológicas podem provocar experiências conscientes diferentes, a depender do contexto [Schachter e Singer 1962, Schachter e Wheeler 1962, Reisenzein 1983].

Diversas abordagens têm sido desenvolvidas para tentar se aproximar ao aspecto cognitivo das emoções usando percepção auditiva, visual, imaginação e/ou simulação de situações ou contextos [Lang e Bradley 2010, Löw et al. 2008, Bradley e Lang 1994, Bradley et al. 2006], com o desenvolvimento de diferentes técnicas para mensurar as respostas dos participantes, usando neuroimagem, mas também a observação do comportamento e a aplicação de instrumentos de avaliação. Tais procedimentos, quando evocam o paradigma do mundo visual, apresentam imagens ou textos com conteúdo que podem misturar a emoção com a experiência particular de um participante por apresentar um conteúdo linguístico com categorias semânticas fortemente arraigadas num contexto sociocultural; podemos, assim, transpor para o estudo de saliência e percepção sociolinguística que o estímulo auditivo ou visual a que um falante é exposto, a depender da variante linguística presente, pode evocar respostas emocionais.

O estudo do processamento da variação linguística tem se valido de medidas de rastreamento ocular, com resultados que evidenciam respostas emocionais após a exposição a um estímulo saliente [Foucart et al. 2019, Boswijk et al. 2020]. Neste texto, apresentamos um estudo exploratório em contextos de exposição a diferentes variantes de uma variável linguística saliente do ponto de vista social, a palatalização progressiva de oclusivas alveolares, a fim de subsidiar o desenvolvimento de técnicas online não invasivas, como o rastreamento ocular, para o estudo do processamento da variação linguística.

\section{Palatalização}

O processo de palatalização no português brasileiro resulta em realizações para /t/ e /d/ em adjacência a /i/: a realização oclusiva alveolar, e realizações palatais ou africadas. 
Em contextos seguidos de /i/, como nas palavras "medida" e "batida" este processo é conhecido como palatalização regressiva. No entanto, quando o contexto é antecedido de /j/, como nas palavras "peito" e "doido", também é possível ocorrer palatalização, em um processo denominado de palatalização progressiva.

A palatalização é um marcador dialetal do português brasileiro, com distribuição relativamente estratificada. Em comunidades em que o processo ainda é incipiente, como é o caso de Sergipe, estudos de produção evidenciam que o perfil de falante associado à variante palatal em contexto de palatalização regressiva é predominante de mulheres, mais jovens e mais escolarizados, residentes na capital. Tem se observado o aumento do uso da variante palatal, que é socialmente bem avaliada. Por outro lado, a variante palatal em ambiente de palatalização progressiva predomina na fala de homens, idosos e menos escolarizados, que residem no interior do estado. A variante palatal está em decréscimo, é socialmente mal avaliada, com comportamento de estereótipo negativo [Souza Neto 2008, Freitag 2015, Freitag e Souza 2016, Freitag e Santos 2016, Ribeiro e Corrêa 2018].

Em um estudo na comunidade considerando o constructo do monitor sociolinguístico [Freitag 2018], as realizações oclusiva e palatal dos dois ambientes de palatalização foram objeto de avaliação. O estudo considerou a exposição dos ouvintesjuízes a sequências de estímulos em áudio de supostas manchetes jornalísticas para um programa de rádio sobre saúde, gravadas por uma falante reconhecida como representativa da comunidade. O comando da tarefa experimental era julgar o grau de profissionalismo de cada um dos treinos de uma estudante de jornalismo candidata à locutora de rádio. Cada sequência de treino foi manipulada para conter diferentes gradientes de palatalização: 100\% (todas palavras-alvo com realização palatal) - 70\% - 30\% - 50\% $30 \%$ - 0\% (todas palavras-alvo com realização oclusiva).

Os ouvintes-juízes deveriam ouvir cada sequência de manchetes e avaliar o grau de profissionalismo do falante dos estímulos. Participaram do estudo falantes sergipanos ( $n=304)$, estratificados quanto a sexo, idade, escolarização e zona de residência.

O resultado mostrou que o processo de palatalização regressiva não é sensível à frequência: com $100 \%$ ou $0 \%$ de realização palatal, a média da nota atribuída ao profissionalismo não apresenta diferença estatisticamente significativa. No entanto, a palatalização progressiva apresenta sensibilidade às frequências da variante [Freitag 2018].

Métodos experimentais (reação subjetiva, matched guise, julgamento de pares mínimos, etc) são usados desde os primeiros estudos da sociolinguística, sempre buscando minimizar os efeitos do Paradoxo do Observador. Em que pese o controle necessário para uma tarefa experimental, o estudo de processamento da variação linguística considera situações autênticas e minimamente invasivas. Nesse sentido, o rastreamento ocular configura-se como uma ferramenta metodológica com potencial para contribuir para a compreensão dos mecanismos cognitivos pelos quais a variação sociolinguística é processada, ao permitir explorar as ligações entre os diferentes níveis da gramática e informações sociais. 


\section{Método}

\subsection{Tarefa e participantes}

O conjunto de dados desta análise foi utilizado em um estudo anterior [Freitag 2018], cujo objetivo era testar o paradigma do monitor sociolinguístico na variação da palatalização de oclusivas em uma variedade do Português Brasileiro. Paralelamente, foi coletada uma amostra em que ao mesmo tempo que os participantes $(n=18)$ eram expostos aos estímulos que consistiam em áudios com diferentes gradações de realização palatal, a tela do computador apresentava o texto escrito, com o objetivo de analisar os movimentos oculares nas zonas críticas, ou seja, as palavras-alvo da manipulação da palatalização. Para esta análise exploratória, consideramos apenas o processo de palatalização progressiva, cujo resultado no estudo de percepção de estímulos auditivos se mostrou sensível à diferença de frequências, sugerindo que o fenômeno é saliente.

\subsection{Rastreio ocular}

Para a coleta dos dados, foi utilizado um aparelho de rastreio ocular marca EyeTribe posicionado na parte inferior da tela do computador que controlava a apresentação dos estímulos, configurado para uma taxa de amostragem de $60 \mathrm{~Hz}$; a resolução da tela foi de $1024 \times 768$ pixels e a distância entre a tela e o participante foi de $57 \mathrm{~cm}$ em média. O software Opensesame [Mathôt et al. 2011] controlou tanto o procedimento de calibragem, que consistiu de 9 pontos, quanto os procedimentos de início e fim do registro do rastreador, assim como a apresentação dos estímulos visuais e auditivos do experimento do monitor sociolinguístico.

Os dados brutos de rastreio foram registrados e analisados usando versões adaptadas dos scripts pyGaze [Dalmaijer et al. 2014], disponíveis como um fork do repositório original no GitHub ${ }^{1}$. Os scripts operacionalizam os movimentos oculares em termos de fixações e sacadas que podem ser agrupadas por áreas de interesse (AdIs), ou áreas retangulares ao redor das quais se espera que se centralizem os movimentos oculares. As medidas obtidas em cada uma das AdIs são o número e a duração das fixações, número e duração das "entradas" nas AdIs, latência da primeira fixação e da primeira entrada. Adicionalmente foram construídos mapas de calor para observar globalmente a distribuição das fixações dentro e além das áreas de interesse.

As AdIs foram definidas como regiões retangulares em torno das palavras-alvo: cuidado, muitos, oito, respeito e muito. O tamanho de cada AdIs incluía, além da palavra em questão, o espaço entre linhas acima e embaixo da mesma, e o espaçamento antes e depois da palavra.

\subsection{Pupilometria}

O rastreador ocular registrou também o tamanho das pupilas dos participantes em séries temporais que foram também extraídas com as versões adaptadas dos scripts de pyGaze [Dalmaijer et al. 2014] e posteriormente processadas no software R [R Development Core Team 2009]. Essas séries representavam a oscilação no tamanho da pupila dos participantes quando observavam e escutavam cada um dos textos.

\footnotetext{
${ }^{1}$ https://github.com/julian-tejada/PyGazeAnalyser
} 
Os dados brutos das séries temporais do tamanho da pupila foram processados aplicando dois filtros: o primeiro para controlar valores outliers na velocidade de dilatação, seguindo a fórmula apresentada por [Kret e Sjak-Shie 2019];

$$
d_{[i]}^{\prime}=\max \left(\left|\frac{d_{[i]}-d_{[i-1]}}{t_{[i]}-t_{[i-1]}}\right|,\left|\frac{d_{[i+1]}-d_{[i]}}{t_{[i+1]}-t_{[i]}}\right|\right)
$$

onde $d_{[i]}$ é o valor do diâmetro da pupila num determinado momento $t_{[i]}$, e a velocidade de dilatação $d_{[i]}^{\prime}$ é calculada como o máximo valor absoluto normalizado entre a mudança relativa ao valor precedente $(i-1)$ e subsequente $(i+1)$. Já o segundo filtro eliminou as piscadas ou valores muito pequenos de tamanho de pupila (pupil size $<=5$ ). Para todos os casos, os valores removidos foram substituídos por interpolação linear, e foram removidos os ensaios nos quais foi preciso interpolar mais do $20 \%$ dos dados.

Uma vez filtradas, as séries temporais foram normalizadas para controlar as diferenças individuais nos tamanhos da pupila. O processo de normalização min-max começou subtraindo de cada valor de cada série o menor valor encontrado na mesma, para posteriormente, dividir cada valor da série pela faixa de valores correspondente [Wendt et al. 2016].

Com as séries normalizadas, procedeu-se a centralizar os diferentes registros em torno do momento no qual cada grupo escutou uma das cinco palavras-alvo (cuidado, muitos, oito, respeito e muito), e usando o pacote ggplot2 [Wickham 2011] estimou-se uma linha de regressão para suavizar a série temporal usando modelos aditivos generalizados seguindo a proposta feita por [Boswijk et al. 2020].

\subsection{Análise de dados}

Os dados de rastreio ocular foram analisados usando modelos de ANOVA de 2 (realização sem palatalização, realização com palatalização) x 5 (cuidado, muitos, oito, respeito e muito) considerando ambos fatores como sendo de medidas repetidas. Quando necessário avaliar as diferenças entre os grupos foi utilizado o pós-teste de Bonferroni. Em todos os casos foram considerados estatisticamente significantes valores de $p<0,05$, e tanto as análises quanto as figuras foram realizadas no $\mathrm{R}$.

\section{Resultados}

\subsection{Rastreio ocular}

Os resultados da calibração mostram que a qualidade dos registros está dentro das margens de erro toleráveis [Ivanchenko et al. 2021], com uma exatidão de $x$ Left $=0,4545 \pm$ 0,33, $x$ Right $=0,4148 \pm 0,29$, yLeft $=0,4545 \pm 0,33, y$ Right $=0,4148 \pm 0,29$ graus, e uma precisão de $X=12,24 \pm 9,21$ e $Y=12,24 \pm 9,21$ pixeis.

Os mapas de calor gerados a partir das frequências de fixações (Figura 1) consideram duas condições: a condição em que os estímulos auditivos das palavras-alvo tinham a realização com palatalização progressiva, que é a variante estigmatizada (a), e a que os estímulos auditivos das palavras-alvo tinham realização oclusiva, padrão, não estigmatizada (b). Dois padrões de fixação podem ser observados: na condição sem palatalização (b), a região que concentra as fixações no mapa de calor se aproxima da região de onde 
anteriormente estava o ponto de fixação que aparecia na tela para iniciar o registro de cada ensaio. Já na condição com palatalização (a), as frequências das fixações concentram-se na margem esquerda do texto, na posição em que as palavras-alvo muito e oito aparecem.

Embora permita a visualização das frequências das fixações, o mapa de calor não permite a comparação dos efeitos entre as condições, o que pode ser realizado com a delimitação das AdIs (Figura 2), que permitem explorar a frequência de entradas (A) e a duração da fixação (B).

A exploração das AdIs mostra que a palavra muitos, nas duas medidas (A e B) e nas duas condições (com palatalização e sem palatalização) foi a que mais captou a atenção dos participantes; mesmo assim, a diferença das medidas nas duas condições é estatisticamente significativa. O mesmo ocorre com as demais palavras-alvo. A diferença entre as condições é maior na medida de duração das fixações do que na frequência das fixações.

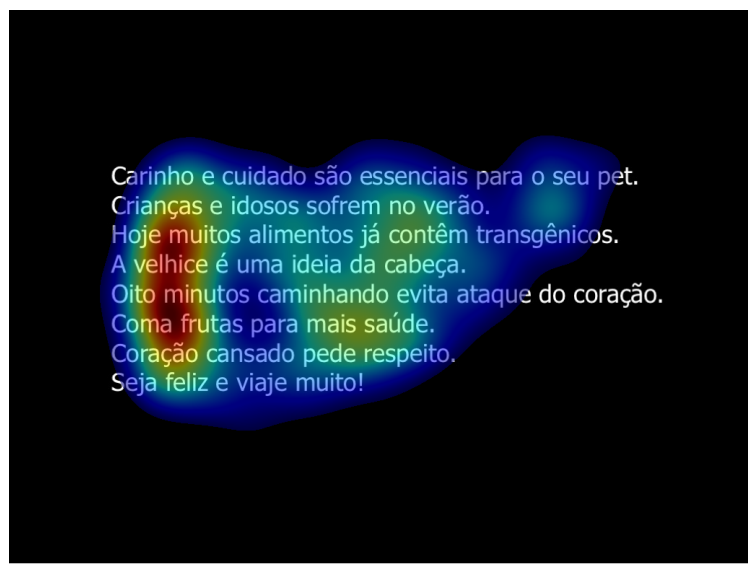

(a)

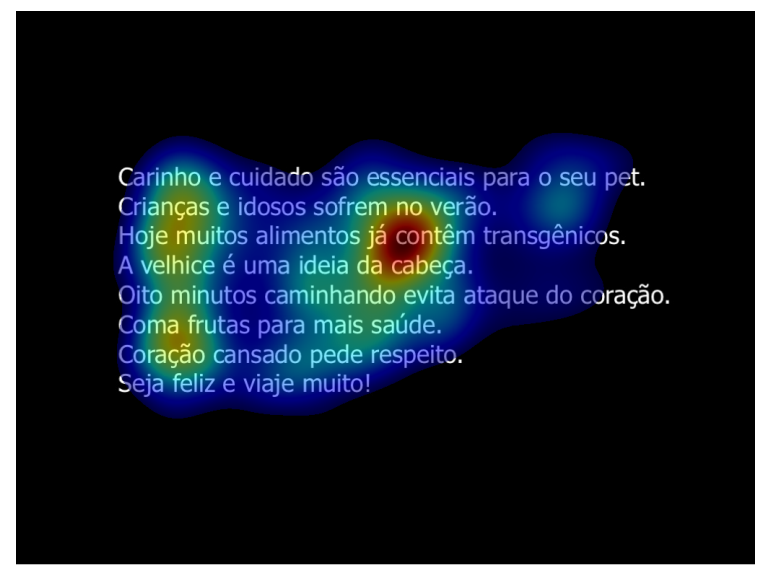

(b)

Figura 1. Mapas de calor gerados a partir das fixações nos estímulos (a) com palatalização e (b) sem palatalização.

\subsection{Pupilometria}

Para a pupilometria, selecionamos as duas palavras-alvo que apresentaram maior diferença nas médias nas duas condições na exploração das AdIs e visualmente presentes nos heatmaps: muitos e oito.

Os dados de mudanças no tamanho da pupila após os participantes terem escutado as palavras-alvo, em suas duas condições, apresentam a mesma dinâmica temporal reportada em estudos que avaliaram a dilatação da pupila como resultado de uma resposta emocional [Partala e Surakka 2003, Oliva e Anikin 2018], com variações que acontecem $400 \mathrm{~ms}$ após ter escutado o estímulo, o que permitem afirmar que houve uma resposta emocional. Na Figura 3 A, observa-se aumento acentuado no diâmetro normalizado da pupila na linha referente à condição com palatalização, evidenciando a dilatação. Em B, a dilatação é ainda mais acentuada. 

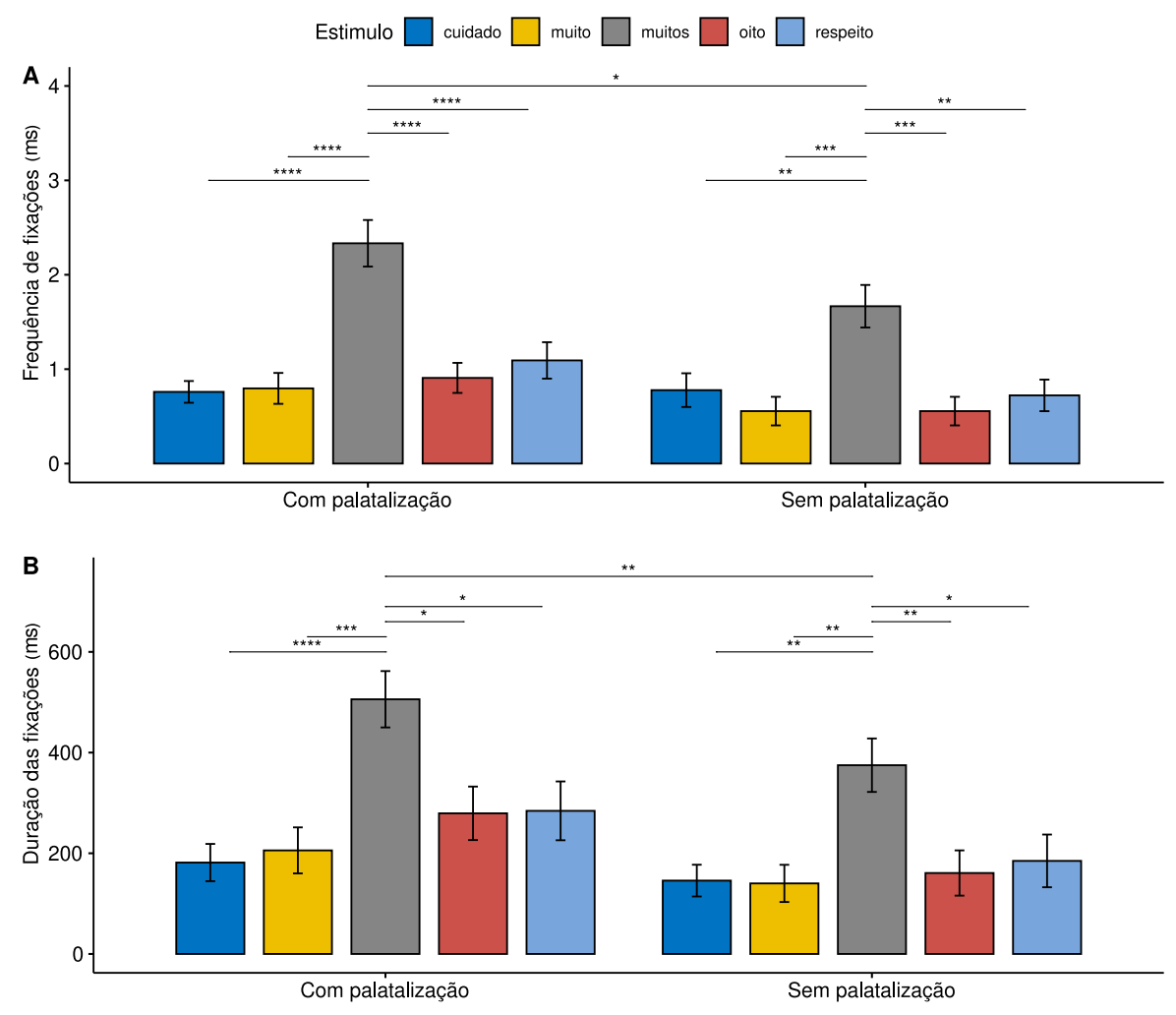

Figura 2. Frequência (A) e duração das fixações (B) em cada uma das AdIs para cada realização

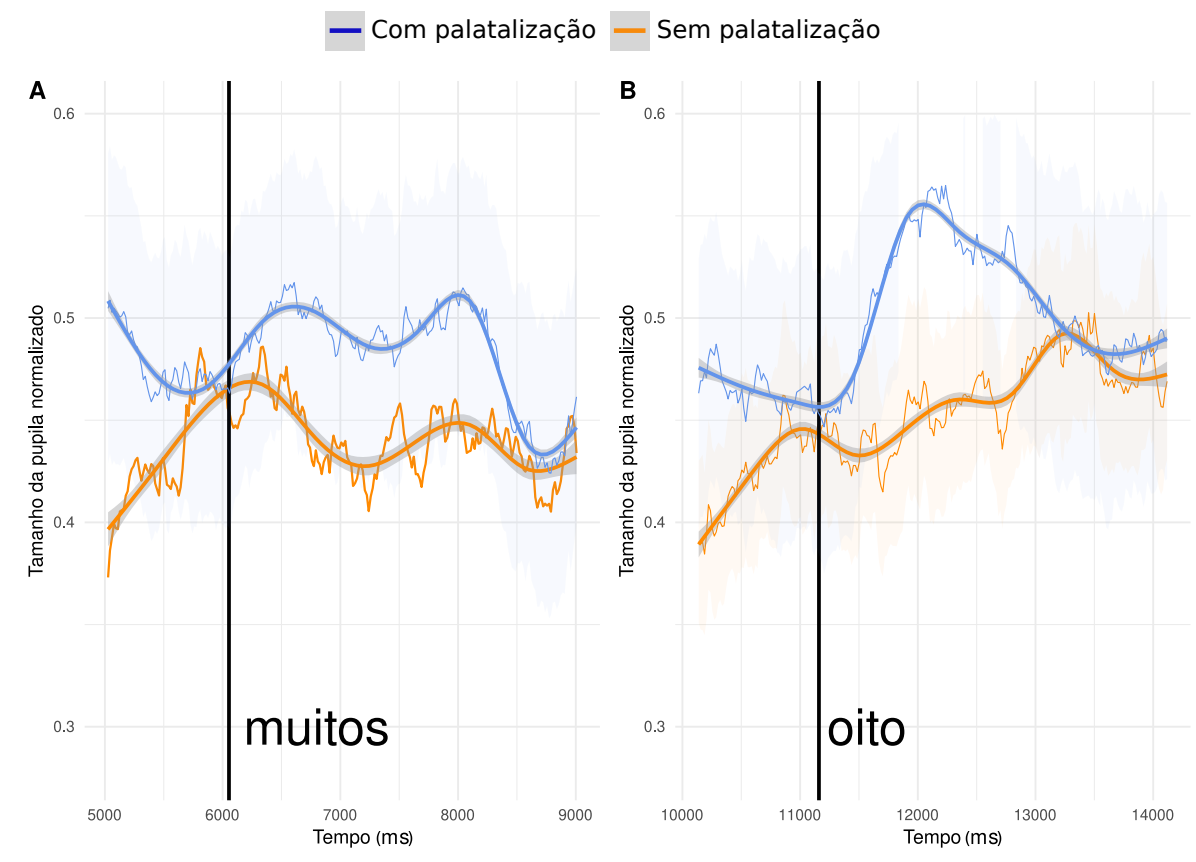

Figura 3. Tamanhos normalizados da pupila dos 18 participantes após terem escutado as palavras (A) muitos e (B) oito nas duas realizações 


\section{Discussão}

O processo de palatalização progressiva é socialmente saliente e marcado, o que é evidenciado pela associação do uso a um perfil de falantes nos estudos de produção, e pelo julgamento negativo atribuído ao uso do traço com palatalização, nos estudos de percepção, configurando-se como uma variável do tipo estereótipo [Labov 1972]. Estereótipos são traços linguísticos que ativam comportamentos estigmatizadores, sendo alvo de preconceito. Assim, é de se esperar que haja uma resposta emocional ante a exposição a um estímulo com este traço.

A análise exploratória dos registros de rastreio ocular evidenciou que a exposição à realização com palatalização captou a atenção (como mostram os resultados do rastreamento ocular, com a medida de duração das fixações) e aumentou a dilatação da pupila (como mostram os resultados da pupilometria), o que podem ser interpretado como evidência de uma resposta emocional.

Estudos têm mostrado que estímulos emocionalmente carregados, sejam visuais ou auditivos, provocam aumento da dilatação da pupila, quando comparados com estímulos neutros [Partala e Surakka 2003, Nakakoga et al. 2020], e essa resposta estaria controlada pelo sistema nervoso autônomo simpático [Bradley et al. 2008], o que permite a utilização da mensuração da mudança na dilatação da pupila como uma medida da resposta emocional ante um determinado estímulo. O estudo de [Boswijk et al. 2020] considerou como alvo diferentes níveis gramaticais, em palavras isoladas; em nosso estudo, consideramos uma única variável, que estava inserida em um contexto linguístico maior (manchetes jornalísticas, cuidadosamente elaboradas para não conter outros traços variáveis salientes para a comunidade de fala onde a tarefa experimental foi realizada). $\mathrm{Na}$ direção apontada por [Boswijk et al. 2020], neste estudo exploratório observamos como uma forma linguística que é inesperada em um contexto (a realização palatal em ambientes de palatalização progressiva por uma universitária candidata à locutora de um programa de rádio), buscando evidenciar a relação entre o traço linguístico e o significado social atribuído pelos falantes. Durante a realização da coleta de dados, comentários informais dos participantes destacavam juízos de valor atribuídos à locutora dos áudios, do tipo "falando desse jeito não pode ser locutora de rádio".

A reação de dilatação da pupila pode ser consequência de diferentes gatilhos, inclusive das diferenças de iluminação, o que exige um ambiente de coleta de dados controlado. Nesse sentido, a coleta foi realizada em uma cabine de gravação, isolando potenciais oscilações de iluminação ou qualquer outra interferência. A diferença na proeminência da curva de aumento do tamanho da pupila entre as palavras-alvo muitos e oito pode ser explicada por um aspecto de frequência do processo de palatalização progressiva: em uma amostra com 10 horas de gravação de fala autêntica [Freitag 2015], foram identificados 775 contextos para palatalização progressiva, dos quais quase metade $(n=360)$ eram da palavra muito e suas flexões. Possivelmente, por conta da frequência, este item tenha um status diferenciado em termos de saliência.

Em relação ao rastreamento ocular, é necessário considerar que não houve planejamento para a disposição das palavras-alvo no texto; para a leitura alfabética, as posições mais à esquerda tendem a ser mais saliente do que a posição à direita; estes aspectos podem ter influenciado a incidência das fixações na palavra-alvo muitos à esquerda, mas não em muito, à direita e no final da linha. 
Apesar das limitações, o estudo exploratório com a palatalização regressiva reforça a potencialidade de utilizar o rastreamento ocular como ferramenta para estabelecer parâmetros para identificação de variáveis socialmente salientes, contribuindo para resolver o problema da literatura com o conceito de "saliência", como mostram [Boswijk et al. 2020].

\section{Referências}

Boswijk, V., Loerts, H., e Hilton, N. H. (2020). Salience is in the eye of the beholder: increased pupil size reflects acoustically salient variables. Ampersand, 7:100061.

Bradley, M. M., Codispoti, M., e Lang, P. J. (2006). A multi-process account of startle modulation during affective perception. Psychophysiology, 43(5):486-497.

Bradley, M. M. e Lang, P. J. (1994). Measuring emotion: The self-assessment manikin and the semantic differential. Journal of Behavior Therapy and Experimental Psychiatry, 25(1):49-59.

Bradley, M. M., Miccoli, L., Escrig, M. A., e Lang, P. J. (2008). The pupil as a measure of emotional arousal and autonomic activation. Psychophysiology, 45(4):602-607.

Dalmaijer, E. S., Mathôt, S., e Van der Stigchel, S. (2014). PyGaze: an open-source, cross-platform toolbox for minimal-effort programming of eyetracking experiments. Behavior Research Methods, 46(4):913-921.

Foucart, A., Santamaría-García, H., e Hartsuiker, R. J. (2019). Short exposure to a foreign accent impacts subsequent cognitive processes. Neuropsychologia, 129:1-9.

Freitag, R., Tejada, J., Brito, Í. D. V., Pinheiro, B., Silva, L. S., Cardoso, P., e Souza, V. R. A. (2020). Estudo piloto da relação entre o julgamento de traços linguísticos e expressões faciais. Cadernos de Linguística, 1(2):01-19.

Freitag, R. M. K. (2015). Socio-stylistic aspects of linguistic variation: schooling and monitoring effects. Acta Scientiarum. Language and Culture, 37(2):127-136.

Freitag, R. M. K. (2018). Saliência estrutural, distribucional e sociocognitiva. Acta scientiarum. Language and culture, 40(2):e41173-e41173.

Freitag, R. M. K. (2020). Effects of the linguistics processing: Palatals in brazilian portuguese and the sociolinguistic monitor. University of Pennsylvania Working Papers in Linguistics, 25(2):4.

Freitag, R. M. K. e Santos, A. d. O. (2016). Percepção e atitudes linguísticas em relação às africadas pós-alveolares em sergipe. A Fala Nordestina: entre a sociolinguística e a dialetologia. São Paulo: Blucher, pages 109-122.

Freitag, R. M. K. e Souza, G. G. A. (2016). O caráter gradiente vs. discreto na palatalização de oclusivas em sergipe. Tabuleiro de Letras, 10(2):78-89.

Ivanchenko, D., Rifai, K., Hafed, Z. M., e Schaeffel, F. (2021). A low-cost, highperformance video-based binocular eye tracker for psychophysical research. Journal of Eye Movement Research, 14(3):10.16910/jemr.14.3.3.

Kecskes, I. (2011). Salience in language production. In Salience and defaults in utterance processing, pages 81-102. De Gruyter Mouton. 
Kerswill, P. e Williams, A. (2011). "salience"as an explanatory factor in language change: evidence from dialect levelling in urban england. In Jones, M. C. and Esch, E., editors, Language Change, pages 81-110. De Gruyter Mounton.

Kret, M. E. e Sjak-Shie, E. E. (2019). Preprocessing pupil size data: Guidelines and code. Behavior Research Methods, 51(3):1336-1342.

Labov, W. (1972). Language in the inner city: Studies in the Black English vernacular. Number 3. University of Pennsylvania Press.

Labov, W., Ash, S., Baranowski, M., Nagy, N., Ravindranath, M., e Weldon, T. (2006). Listeners' sensitivity to the frequency of sociolinguistic variables. University of Pennsylvania Working Papers in Linguistics, 12(2):10.

Labov, W., Ash, S., Ravindranath, M., Weldon, T., Baranowski, M., e Nagy, N. (2011). Properties of the sociolinguistic monitor. Journal of Sociolinguistics, 15(4):431-463.

Lang, P. J. e Bradley, M. M. (2010). Emotion and the motivational brain. Biological Psychology, 84(3):437-450.

Levon, E. e Fox, S. (2014). Social salience and the sociolinguistic monitor: A case study of ing and th-fronting in britain. Journal of English Linguistics, 42(3):185-217.

Löw, A., Lang, P. J., Smith, J. C., e Bradley, M. M. (2008). Both predator and prey. Psychological Science, 19(9):865-873.

Mathôt, S., Schreij, D., e Theeuwes, J. (2011). OpenSesame: An open-source, graphical experiment builder for the social sciences. Behavior Research Methods, 44(2):314324.

Nakakoga, S., Higashi, H., Muramatsu, J., Nakauchi, S., e Minami, T. (2020). Asymmetrical characteristics of emotional responses to pictures and sounds: Evidence from pupillometry. PLOS ONE, 15(4):e0230775.

Oliva, M. e Anikin, A. (2018). Pupil dilation reflects the time course of emotion recognition in human vocalizations. Scientific Reports, 8(1):4871.

Partala, T. e Surakka, V. (2003). Pupil size variation as an indication of affective processing. International Journal of Human-Computer Studies, 59:185-198.

R Development Core Team (2009). R: A Language and Environment for Statistical Computing. R Foundation for Statistical Computing, Vienna, Austria. ISBN 3-900051-070.

Reisenzein, R. (1983). The schachter theory of emotion: Two decades later. Psychological bulletin, 94(2):239.

Ribeiro, C. C. d. S. e Corrêa, B. T. R. d. A. (2018). Avaliação social da palatalização de/t, d/em sergipe. A Cor das Letras, 19(4Especial):109-123.

Schachter, S. e Singer, J. (1962). Cognitive, social, and physiological determinants of emotional state. Psychological review, 69(5):379.

Schachter, S. e Wheeler, L. (1962). Epinephrine, chlorpromazine, and amusement. The Journal of Abnormal and Social Psychology, 65(2):121.

Souza Neto, A. F. d. (2008). Realizações dos fonemas/t/e/d/em aracaju sergipe. 
Squires, L. (2016). Processing grammatical differences: Perceiving versus noticing. In Babel, A., editor, Awareness and control in sociolinguistic research, pages 80-103. Cambridge.

Wendt, D., Dau, T., e Hjortkjær, J. (2016). Impact of Background Noise and Sentence Complexity on Processing Demands during Sentence Comprehension. Frontiers in Psychology, 7:345.

Wickham, H. (2011). ggplot2. WIREs Computational Statistics, 3(2):180-185. _eprint: https://wires.onlinelibrary.wiley.com/doi/pdf/10.1002/wics.147. 